\title{
FLORÍSTICA E ESTRUTURA DA VEGETAÇÃO ARBUSTIVO-ARBÓREA DO SUB-BOSQUE DE UM POVOAMENTO DE Eucalyptus grandis W. Hill ex Maiden EM VIÇOSA, MG, BRASIL ${ }^{1}$
}

Priscila Bezerra de Souza ${ }^{2}$, Sebastião Venâncio Martins ${ }^{3}$, Sumami Rebonato Costalonga ${ }^{4}$ e Grasiely de Oliveira Costa ${ }^{5}$

\begin{abstract}
RESUMO - Este estudo teve como objetivo conhecer a composição florística e a estrutura da vegetação arbustivoarbórea no sub-bosque de povoamento de Eucalyptus grandis na Reserva Florestal da Mata do Paraíso, em Viçosa, MG. Foram demarcadas 40 parcelas contíguas de 5 x $5 \mathrm{~m}$, dispostas em transectos de 5 x $50 \mathrm{~m}$, nas quais foram medidos, identificados e classificados quanto às síndromes de dispersão de sementes e às categorias sucessionais. Foram amostrados 884 indivíduos pertencentes a 50 espécies e 22 famílias. As espécies que se destacaram em valor de importância foram Psychotria sessilis, Siparuna guianensis e Erythroxylum pelleterianum, principalmente com relação à elevada densidade. Predominaram em densidade espécies secundárias tardias com síndromes de dispersão zoocórica. A riqueza florística encontrada pode ser considerada alta, por se tratar de sub-bosque de Eucalyptus grandis, e reflete o potencial da utilização dessa espécie como catalisadora de vegetação arbustivo-arbórea nativa em áreas degradadas.
\end{abstract}

Palavras-chave: Eucalyptus grandis, floresta catalisadora e sub-bosque.

\section{FLORISTIC AND STRUCTURE OF TREE-SHRUB VEGETATION IN UNDERSTORY OF Eucalyptus grandis W. Hill ex Maiden STANDS, IN VIÇOSA, MG, BRAZIL}

\begin{abstract}
The objective of the present study was to analyze the floristic composition and structure of treeshrub vegetation in understory of Eucalyptus grandis W. former Hill Maiden, Paraíso Forest Reserve, Viçosa, MG. Forty adjacent $5 \times 5 \mathrm{~m}$ plots were demarcated and arranged in $5 \times 50 \mathrm{~m}$ transects, in which individuals were measured, identified and classified in relation to seed dispersal syndromes and successional categories. Eight hundred and eighty four individuals belonging to 50 species and 22 families were recorded. The species with the highest Importance Value were Psychotria sessilis, Siparuna guianensis and Erythroxylum pelleterianum, particularly with regard to high density. Late secondary species with zoochorous dispersal syndromes prevailed in density. The founded floristic richness can be considered high for a Eucalyptus grandis understory and reflects the potential for using the species as catalyst for native tree-shrub vegetation in degraded areas.
\end{abstract}

Keywords: Eucalyptus grandis, forest catalyst and understory.

\section{INTRODUÇÃO}

Diversos estudos têm mostrado que plantações homogêneas florestais podem acelerar o processo de sucessão florestal em áreas degradadas, catalisando plantas nativas em seus sub-bosques (PARROTA et al., 1997; POWERS et al., 1997; GELDENHUYS, 1997; NAPPO et al., 2000). Muitas espécies arbóreas utilizadas nos reflorestamentos comerciais conseguem crescer rapidamente em solos de baixa fertilidade, comuns em áreas degradadas, e podem fornecer condições favoráveis para a regeneração de espécies nativas, como poleiros e abrigo para aves dispersoras de sementes e sombra para plântulas de espécies tardias na sucessão.

\footnotetext{
${ }^{1}$ Recebido em 10.07.2006 e aceito para publicação em 05.02.2007.

${ }^{2}$ Programa de Pós-Graduação em Botânica da UFV. E-mail: <pri_ufv@ yahoo.com.br>.

${ }^{3}$ Departamento de Engenharia Florestal da Universidade Federal de Viçosa, MG. E-mail: <venancio@ufv.br>.

${ }^{4}$ Mestre em Ciência Florestal. E-mail: <sumami_su@yahoo.com.br>.

${ }^{5}$ Bióloga. E-mail: <grasielycosta@yahoo.com.br>.
} 
Plantios homogêneos de eucalipto, introduzidos por programas de fomento e atualmente em fase de exploração, apresentam significativo desenvolvimento do sub-bosque, indicando um processo de sucessão favorável à recuperação da biodiversidade. Essa situação evidencia possibilidades para a condução de povoamentos auxiliares com espécies florestais nativas, favorecendo a recuperação de áreas degradadas, controle de pragas, conservação do solo e manutenção da fauna. O sub-bosque de povoamentos homogêneos, até então visto como grande entrave por prejudicar o desenvolvimento das espécies de interesse econômico, aumentar o risco de incêndios e dificultar as operações de manejo, combate às formigas e exploração, começa a ser valorizado em seu aspecto de biodiversidade, concorrendo para a estabilidade ambiental (FAO, 1987; LIMA, 1993).

Numa paisagem desprovida de cobertura florestal, como pastagens abandonadas, a sucessão secundária pode ser extremamente lenta, já que esse processo ecológico depende de alguns fatores, como a chuva de sementes, a germinação de sementes, o microclima e o solo (HOLL, 1999). Nesse contexto, florestas plantadas podem criar um ambiente favorável à chegada de sementes e ao estabelecimento de plântulas de espécies nativas em seus sub-bosques, ao fornecerem sombra para espécies tolerantes e poleiros para aves e morcegos dispersores de sementes de remanescentes florestais das vizinhanças. Como resultado, tais florestas podem, em médio e longo prazos, apresentar um sub-bosque rico em espécies arbustivo-arbóreas nativas (SCHILITTLER, 1984; CALEGARIO et al., 1993; SILVA JÚNIOR et al., 1995; NAPPO et al., 2000; NERI et al., 2005). Assim, na sucessão secundária em áreas degradadas, árvores plantadas de espécies exóticas podem ter funções semelhantes ou próximas à de árvores nativas remanescentes, como verificado na literatura (GUEVARA et al., 1986; RODRIGUES et al., 2004).

A utilização de florestas plantadas com espécies exóticas de rápido crescimento, como alternativa de restauração florestal, apresenta como vantagens a rusticidade dessas espécies, que podem crescer em solos pobres e degradados, controlando processos erosivos, e a possibilidade de utilização da madeira após a formação de sub-bosque por espécies nativas. Além disso, o sub-bosque dos reflorestamentos pode servir de fonte de mudas de espécies arbustivo-arbóreas para transplantes em outras áreas degradadas.
Assim, esta pesquisa propôs avaliar a hipótese de que o plantio de espécies de rápido crescimento como Eucalyptus grandis tende a promover (catalisar) a regeneração do sub-bosque com espécies arbustivoarbóreas nativas das formações florestais da região de abrangência deste estudo, sendo essa uma forma de acelerar os processos de sucessão secundária em áreas degradadas. Portanto, esta pesquisa objetivou conhecer a composição florística e a estrutura da vegetação arbustivo-arbórea no sub-bosque de povoamento de Eucalyptus grandis, com idade de aproximadamente 35 anos, na Reserva Florestal da Mata do Paraíso, em Viçosa, MG.

\section{MATERIAL E MÉTODOS}

O estudo foi desenvolvido no sub-bosque de um povoamento de Eucalyptus grandis W. Hill ex Maiden de aproximadamente 2 ha inserido na Reserva Florestal Mata do Paraíso (2048'07'S e 4251'31'W), no Município de Viçosa, MG. Esse povoamento foi implantado na década de 1970 , no espaçamento 3,0 $\mathrm{x} 2,0 \mathrm{~m}$, e encontra-se nas proximidades de remanescente de florestas nativas.

A Reserva Florestal Mata do Paraíso pertence à Universidade Federal de Viçosa e possui 195 ha de área, com altitudes variando de 690 a 800 m (BRAZ et al., 2002). A vegetação da reserva é composta por trechos de Floresta Estacional Semidecidual (VELOSO et al., 1991) e compõe um mosaico de diferentes estágios sucessionais, pequenas áreas de brejo (SILVA JÚNIOR et al., 2004), trechos de pastagem abandonada e talhões abandonados de Eucalyptus grandis e Pinus sp.

O clima na região é do tipo Cwb (Köppen), mesotérmico com verões quentes e chuvosos e invernos frios e secos. A temperatura média anual é de $21,8^{\circ} \mathrm{C}$ e a precipitação pluviométrica média anual, de 1.314,2 mm (CASTRO et al., 1983).

A vegetação do sub-bosque do povoamento de Eucalyptus grandis foi avaliada quantitativamente em outubro de 2004, utilizando-se o método de parcelas (MUELLER-DOMBOIS e ELLENBERG, 1974). Foram instaladas 40 parcelas de $5 \times 5 \mathrm{~m}$, em quatro transectos paralelos de $5 \times 50 \mathrm{~m}$. A distribuição dos transectos foi feita sistematicamente a cada $5 \mathrm{~m}$ da borda do talhão. Nessas parcelas foram amostrados todos os indivíduos arbustivo-arbóreos com altura igual ou superior $1 \mathrm{~m}$. Desses indivíduos foram tomadas medidas de diâmetro 
ao nível do solo e altura total, bem como coletado material botânico para identificação. A identificação taxonômica foi realizada através de comparações com material do Herbário VIC da Universidade Federal de Viçosa, literatura especializada e consultas a especialistas, quando necessário. Para atualização dos binômios específicos foram utilizados o índice de espécies Royal Botanic of Kew (1993) e o site do Missouri Botanical Garden (disponível em: http://www.mobot.org/W3T/search/ vast.html), no mês de junho de 2006. O sistema de classificação adotado foi o Botânica Sistemática, baseado em APG II (SOUZA e LORENZI, 2005).

Foram calculados os parâmetros usuais em fitossociologia (densidade absoluta e relativa, freqüência absoluta e relativa, dominância absoluta e relativa, valor de importância) (MUELLER-DOMBOIS e ELLENBERG, 1974). Para analisar a diversidade de espécies, foram utilizados os índices de diversidade de Shannon (H') e a eqüabilidade de Pielou (J') (BROWER e ZAR, 1984). As análises foram realizadas com o programa FITOPAC (SHEPHERD, 1994).

As espécies amostradas foram classificadas quanto à síndrome de dispersão de sementes em zoocóricas, autocóricas e anemocóricas (van der PIJL, 1982; MORELLATO e LEITÃO FILHO, 1992) e à categoria sucessional em pioneiras, secundárias iniciais e secundárias tardias, seguindo-se os trabalhos de Gandolfi et al. (1995), Bernacci e Leitão Filho (1996), Martins e Rodrigues (2002) e Martins et al. (2004).

\section{RESULTADOS E DISCUSSÃO}

No Quadro 1, encontra-se à relação das espécies arbustivas e arbóreas amostradas no sub-bosque de um povoamento de Eucalyptus grandis com idade aproximada de 35 anos, implantado no Município de Viçosa, MG. As espécies foram listadas em ordem alfabética de família botânica, nomes genéricos e específicos, além da classificação quanto ao grupo ecológico e tipo de síndrome de dispersão de diásporos.

Foram amostrados 884 indivíduos arbustivoarbóreos (845 vivos e 39 mortos), sendo os vivos pertencentes a 22 famílias, 38 gêneros e 50 espécies. A densidade total estimada por hectare foi de 1.768 indivíduos e a área basal, de 3,46 m². Essa riqueza de espécie é superior à encontrada em outros estudos realizados em sub-bosque de Eucalyptus, que foi de 25 espécies em Assis, SP (DURIGAN et al. 1997), 40 em Bom Despacho, MG (SAPORETTI JR. et al., 2003) e 47 em Paraopeba, MG (NERI et al., 2005). Isso pode ser atribuído à maior proximidade de fontes de propágulos no talhão de eucalipto em estudo, uma vez que este se encontra no interior de uma reserva de Floresta Estacional Semidecidual.

Quadro 1 - Lista das espécies amostradas em sub-bosque de Eucalyptus grandis em Viçosa-MG, ordenadas alfabeticamente por famílias, subfamílias e espécies, com as respectivas categorias sucessionais (CS), pioneira (P), secundária inicial (Si) e secundária tardia (St), ou não-classificada (Nc); e síndromes de dispersão (SD), zoocórica (Zoo) e anemocórica (Ane)

Table 1 - List of sampled species in understory of Eucalyptus grandis in Viçosa, MG, in alphabetical order of families, subfamilies and species; with the respective successional category (CS), pioneer (P), initial secondary (Si), late secondary (St) and unclassified (Nc); and seed dispersal syndromes Zoo (zoochorous dispersal) and Ane (anemochorous dispersal)

\begin{tabular}{|c|c|c|c|}
\hline Famílias/Subfamílias/Espécies & $\mathrm{CS}$ & Nome Popular & SD \\
\hline $\begin{array}{l}\text { Anacardiaceae } \\
\text { Tapirira obtusa (Benth.) D.J. Mitch. }\end{array}$ & $\mathrm{Si}$ & Camanducaia & Zoo \\
\hline $\begin{array}{l}\text { Annonaceae } \\
\text { Guatteria sellowiana } \text { Schltdl. }\end{array}$ & $\mathrm{Si}$ & Pindaíba-negra & Zoo \\
\hline Rollinia laurifolia Schltdl. & $\mathrm{Si}$ & Araticum & Zoo \\
\hline Xylopia sericea A. St.-Hil. & $\mathrm{Si}$ & Banana-de-macaco & Zoo \\
\hline $\begin{array}{l}\text { Apocynaceae } \\
\text { Aspidosperma subincanum Mart. } \\
\text { Aquifoliaceae }\end{array}$ & St & Perobinha & Ane \\
\hline Ilex brevicuspis Reissek & $\mathrm{Nc}$ & Voadeira & Zoo \\
\hline $\begin{array}{l}\text { Asteraceae } \\
\text { Vernonia diffusa Less. }\end{array}$ & $\underline{P}$ & Pau-fumo & Ane \\
\hline
\end{tabular}


Quadro 1 - Cont.

Table 1 - Cont.

\begin{tabular}{|c|c|c|c|}
\hline Famílias/Subfamílias/Espécies & $\mathrm{CS}$ & Nome Popular & SD \\
\hline \multicolumn{4}{|l|}{ Bignoniaceae } \\
\hline Jacaranda macrantha Cham. & $\mathrm{Si}$ & Jacarandá & Ane \\
\hline Sparattosperma leucanthum (Vell.) K. Schum. & $\mathrm{Si}$ & Caroba-branca & Ane \\
\hline \multicolumn{4}{|l|}{ Fabaceae-Caesalpinioideae } \\
\hline Apuleia leiocarpa (Vogel) J.F. Macbr. & $\mathrm{Si}$ & Guajuvira & Ane \\
\hline Bauhinia forficata Link & $\mathrm{P}$ & Pata-de vaca & Ane \\
\hline Copaifera langsdorffii Desf. & St & Copaíba & Zoo \\
\hline Peltophorum dubium (Spreng.) Taub. & $\mathrm{Si}$ & Canjica & Ane \\
\hline Machaerium aculeatum Raddi & $\mathrm{Si}$ & Jacarandá de espinho & Ane \\
\hline Machaerium nyctitans (Vell.) Benth. & $\mathrm{Si}$ & Jacarandá-ferro & Ane \\
\hline \multicolumn{4}{|l|}{ Fabaceae-Mimosoideae } \\
\hline Anadenanthera colubrina (Vell.) Brenan & $\mathrm{Si}$ & Angico branco & Ane \\
\hline Inga sessilis (Vell.) Mart. & $\mathrm{Si}$ & Ingá & Zoo \\
\hline Piptadenia gonoacantha (Mart.) J.F. Macbr. & $\mathrm{Si}$ & Pau-jacaré & Ane \\
\hline \multicolumn{4}{|l|}{ Clusiaceae } \\
\hline Rheedia gardneriana Planch. \& Triana & $\mathrm{St}$ & Bacupari & Zoo \\
\hline Vismia brasiliensis Choisy & $\mathrm{P}$ & Pão-de-lacre & Zoo \\
\hline \multicolumn{4}{|l|}{ Erythroxylaceae } \\
\hline Erythroxylum citrifolium A. St.-Hil. & $\mathrm{Si}$ & Pimenta de nambú & Zoo \\
\hline Erythroxylum pelleterianum A. St.-Hil. & $\mathrm{Si}$ & - & Zoo \\
\hline \multicolumn{4}{|l|}{ Euphorbiaceae } \\
\hline Alchornea glandulosa Poep. \& Endl. & $\mathrm{P}$ & Tanheiro & Zoo \\
\hline \multicolumn{4}{|l|}{ Flacourtiaceae } \\
\hline Carpotroche brasiliensis (Raddi) A. Gray & St & Sapucainha & Zoo \\
\hline Casearia arborea (Rich.) Urb. & $\mathrm{Si}$ & Pau-espeto & Zoo \\
\hline Casearia decandra Jacq. & St & Guaçatonga & Zoo \\
\hline \multicolumn{4}{|l|}{ Lauraceae } \\
\hline Nectandra oppositifolia Nees \& Mart. & $\mathrm{Si}$ & Canela-garuva & Zoo \\
\hline Ocotea corymbosa (Meisn.) Mez & St & Canela-bosta & Zoo \\
\hline Ocotea difusa Van der Werff & $\mathrm{Si}$ & Canelinha & Zoo \\
\hline Ocotea odorifera (Vellozo) Rohwer & $\mathrm{St}$ & Canela-cheirosa & Zoo \\
\hline \multicolumn{4}{|l|}{ Malvaceae } \\
\hline Pseudobombax grandiflorum (Cav.) A. Robyns & $\mathrm{P}$ & Embiriçu & Ane \\
\hline \multicolumn{4}{|l|}{ Melastomataceae } \\
\hline Miconia chamissois Naudin & $\mathrm{P}$ & Sabiazeira & Zoo \\
\hline Miconia rigidiuscula Cogn. & $\mathrm{P}$ & Capa-rosa & Zoo \\
\hline \multicolumn{4}{|l|}{ Meliaceae } \\
\hline Cabralea canjerana (Vell.) Mart. & St & Canjerana & Zoo \\
\hline Trichilia pallida $\mathrm{Sw}$. & St & Carrapeta & Zoo \\
\hline Trichilia lepidota Mart. & $\mathrm{St}$ & Baga-de-morcego & Zoo \\
\hline \multicolumn{4}{|l|}{ Siparunaceae } \\
\hline Siparuna guianensis Aubl. & St & Marinheiro & Zoo \\
\hline Siparuna reginae (Tul.) A. DC. & $\mathrm{St}$ & - & Zoo \\
\hline \multicolumn{4}{|l|}{ Myrtaceae } \\
\hline Myrcia fallax (A. Richard) DC. & $\mathrm{Si}$ & Guamirim-do-preto & Zoo \\
\hline Myrcia rostrata DC. & $\mathrm{P}$ & Guamirim-de-folha-miúda & Zoo \\
\hline \multicolumn{4}{|l|}{ Piperaceae } \\
\hline Piper gaudichaudianum Kunth & $\mathrm{P}$ & Jaguarandi & Zoo \\
\hline \multicolumn{4}{|l|}{ Rubiaceae } \\
\hline Psychotria longepedunculata (Gardner) Müll. Arg. & St & Café da mata & Zoo \\
\hline Psychotria nuda (Cham. \& Schltdl.) Wawra & $\mathrm{St}$ & Grandiúva d'anta & Zoo \\
\hline Psychotria sessilis (Vell.) Müll. Arg. & St & - & Zoo \\
\hline
\end{tabular}

R. Árvore, Viçosa-MG, v.31, n.3, p.533-543, 2007 
Quadro 1-Cont.

Table 1 - Cont.

\begin{tabular}{llcl}
\hline Famílias/Subfamílias/Espécies & $\mathrm{CS}$ & Nome Popular & SD \\
\hline Rutaceae & & & Ane \\
Dictyoloma incanescens DC. & $\mathrm{Si}$ & Zoo \\
Zanthoxylum rhoifolium Lam. & $\mathrm{Si}$ & Mamica-de-cadela & Zoo \\
Sapindaceae & & & Zoo \\
Allophylus edulis (A. St.-Hil., Cambess. \& A. Juss.) Radlk. & $\mathrm{P}$ & $\mathrm{Si}$ & Zoo \\
Cupania vernalis Cambess. & $\mathrm{Si}$ & Maria-preta & - \\
$\begin{array}{l}\text { Matayba elaeagnoides Radlk. } \\
\text { Solanaceae }\end{array}$ & $\mathrm{Si}$ & Zoo \\
Solanum cernuum Vell. & $\mathrm{P}$ & Braço-de-mono & \\
\hline
\end{tabular}

A classificação sucessional das espécies (Quadro 2) revelou maior riqueza de espécies iniciais na sucessão secundária, com o grupo formado pelas pioneiras e secundárias iniciais, totalizando $68 \%$ das espécies. Entretanto, quando se considerou o número de indivíduos vivos, foi verificado que as espécies secundárias tardias somaram 59,17\% do total amostrado. Esses resultados apontaram que o povoamento de Eucalyptus grandis tem fornecido condições ecológicas, como sombra, para a catalisação de espécies dos diferentes grupos sucessionais e que espécies finais da sucessão estão conseguindo se destacar em densidade nesse ambiente sombreado.

O índice de diversidade especifica de Shannon (H') foi de 2,89 e a eqüabilidade (J), de 0,74. Em levantamentos realizados especificamente com vegetação remanescente de sub-bosque em Viçosa, MG, os valores de H' têm sido bastante variáveis, refletindo o estádio sucessional dessas florestas. Assim, nessa mesma reserva foram obtidos valores de H' de 1,91 para o sub-bosque de um trecho de floresta secundária inicial e de 3,15 para trecho de floresta madura sem intervenção antrópica nos últimos 40 anos (SILVA JÚNIOR et al., 2004). No sub-bosque de povoamento homogêneo de Mimosa scabrella Benth. em Poços de Caldas, MG, foi estimado um valor de diversidade de 2,85 (NAPPO et al., 2000), enquanto no sub-bosque de povoamento de Eucalyptus saligna em Itatinga, SP, foram estimados valores de 2,51 e 3,75 (SARTORI et al., 2002). Portanto, pode-se considerar que a diversidade do sub-bosque avaliado está refletindo uma condição similar à do sub-bosque de florestas semidecíduas secundárias da região e do sub-bosque de povoamentos de florestas plantadas em outras localidades do Sudeste brasileiro.

As famílias mais ricas foram Fabaceae
(Leguminosae), com nove espécies, seguida por Lauraceae (4), Rubiaceae, Meliaceae, Flacourtiaceae e Sapindaceae, com três espécies cada. Dentre as Fabaceae, quatro são Fabaceae-Caesalpinioideae, três FabaceaeMimosoideae e duas Fabaceae-Faboideae (Quadro 3). As famílias com maior número de indivíduos foram Rubiaceae (264), Siparunaceae (153), Melastomataceae (61), Erythroxylaceae (60), Fabaceae-Mimosoideae (40), Flacourtiaceae (32), Lauraceae (31) e Meliaceae (29). Essas oito famílias, juntas, contribuíram com $75,8 \%$ do número total de indivíduos amostrados.

O destaque de Fabaceae em riqueza de espécies já era esperado, uma vez que essa família é característica de florestas semidecíduas e tem se destacado na maioria dos levantamentos realizados nessa formação (MEIRA NETO et al., 2000; RIBAS et al., 2003; SILVA et al., 2003; ARAÚJO et al., 2005). Além disso, a alta densidade das leguminosas pode ser atribuída à capacidade de fixação biológica de nitrogênio de muitas espécies dessa família, facilitando a regeneração em solos pobres e degradados (CARVALHO, 1998; CAMPELLO, 1998).

Quadro 2 - Proporção de espécies e indivíduos da vegetação arbustivo-arbórea avaliada em sub-bosque de Eucalyptus grandis em Viçosa, MG, conforme as categorias sucessionais

Table 2 - Proportion of species and individuals of the shrubtree vegetation sampled in understory of Eucalyptus grandis in Viçosa, MG, according to the successional categories

\begin{tabular}{lccccc}
\hline Categoria & \multicolumn{3}{c}{ Espécies } & & \multicolumn{2}{c}{ Indivíduos } \\
\cline { 2 - 3 } \cline { 6 - 6 } sucessional & Quantidade & $\%$ & & Quantidade & $\%$ \\
\hline Pioneira & 11 & 22 & & 135 & 15,98 \\
Secundária inicial & 23 & 46 & & 205 & 24,26 \\
Secundária tardia & 15 & 30 & & 500 & 59,17 \\
Não classificadas & 1 & 2 & & 5 & 0,59 \\
\hline Total & 50 & 100 & 845 & 100 \\
\hline
\end{tabular}

R. Árvore, Viçosa-MG, v.31, n.3, p.533-543, 2007 
Quadro 3 - Estimativa dos parâmetros fitossociológicos das famílias e subfamílias amostradas em sub-bosque de Eucalyptus grandis em Viçosa, MG, ordenadas de forma decrescente em valor de importância, em que: D - densidade absoluta, $\mathrm{N}$ - número de espécies, \% sp - porcentagem de espécies, DR - densidade relativa (\%), DoA - dominância absoluta (\%), DoR - dominância relativa (\%), FA - freqüência absoluta (\%), FR - frequiência relativa (\%) e VI - valor de importância (\%)

Table 3 - Estimate of phytosociological parameters of families and sub-families sampled in understory of Eucalyptus grandis in Viçosa, MG, in descending order of importance value, where DA - absolute density, $N$ - species number, \%Sp - species percentage, DR - relative density (\%), DoA - absolute dominance (\%), DoR - relative dominance (\%), $F A$ - absolute frequency (\%), FR - relative frequency (\%), VI-importance value (\%)

\begin{tabular}{|c|c|c|c|c|c|c|c|c|c|}
\hline Família/Subfamília & $\mathrm{DA}$ & $\mathrm{N}$ & $\% \mathrm{Sp}$ & DR & DoA & DoR & FA & FR & VI \\
\hline Rubiaceae & 264 & 3 & 5,88 & 29,86 & 0,85 & 24,76 & 100 & 11,68 & 22,1 \\
\hline Siparunaceae & 153 & 2 & 3,92 & 17,31 & 0,68 & 19,81 & 100 & 11,42 & 16,17 \\
\hline Erythroxylaceae & 60 & 2 & 3,92 & 6,79 & 0,30 & 8,87 & 60 & 7,41 & 7,68 \\
\hline Melastomataceae & 61 & 2 & 3,92 & 6,98 & 0,17 & 4,92 & 60 & 6,55 & 6,12 \\
\hline Lauraceae & 31 & 4 & 7,84 & 3,51 & 0,12 & 3,45 & 40 & 5,13 & 4,03 \\
\hline Clusiaceae & 24 & 2 & 3,92 & 2,71 & 0,13 & 3,91 & 50 & 5,41 & 4,01 \\
\hline Flacourtiaceae & 32 & 3 & 5,88 & 3,62 & 0,092 & 2,64 & 50 & 5,71 & 3,98 \\
\hline Annonaceae & 25 & 2 & 3,92 & 2,83 & 0,14 & 4,24 & 40 & 4,84 & 3,95 \\
\hline F. Mimosoideae & 40 & 3 & 5,88 & 4,52 & 0,05 & 1,64 & 50 & 5,7 & 3,95 \\
\hline F. Caesalpinioideae & 22 & 4 & 7,84 & 2,49 & 0,10 & 3,07 & 40 & 4,84 & 3,46 \\
\hline Euphorbiaceae & 23 & 1 & 1,96 & 2,67 & 0,10 & 3,15 & 40 & 4,56 & 3,43 \\
\hline Meliaceae & 29 & 3 & 5,88 & 3,28 & 0,10 & 2,94 & 20 & 2,85 & 3,01 \\
\hline Bignoniaceae & 13 & 2 & 3,92 & 1,47 & 0,08 & 2,44 & 30 & 3,13 & 2,33 \\
\hline Solanaceae & 12 & 1 & 1,96 & 1,36 & 0,02 & 0,68 & 20 & 2,56 & 1,53 \\
\hline Myrtaceae & 9 & 2 & 3,92 & 1,02 & 0,02 & 0,83 & 20 & 2,56 & 1,47 \\
\hline Sapindaceae & 13 & 3 & 5,88 & 1,47 & 0,01 & 0,55 & 10 & 1,71 & 1,24 \\
\hline Piperaceae & 7 & 1 & 1,96 & 0,79 & 0,03 & 0,89 & 10 & 1,42 & 1,03 \\
\hline Rutaceae & 4 & 2 & 3,92 & 0,45 & 0,03 & 1,01 & 10 & 1,14 & 0,86 \\
\hline Anacardiaceae & 7 & 2 & 3,92 & 0,79 & 0,00 & 0,24 & 10 & 1,42 & 0,82 \\
\hline Aquifoliaceae & 5 & 1 & 1,96 & 0,57 & 0,02 & 0,74 & 10 & 0,85 & 0,72 \\
\hline Apocynaceae & 4 & 1 & 1,96 & 0,45 & 0,02 & 0,74 & 10 & 0,85 & 0,69 \\
\hline Malvaceae & 3 & 1 & 1,96 & 0,34 & 0,02 & 0,76 & 10 & 0,85 & 0,65 \\
\hline F. Faboideae & 3 & 2 & 3,92 & 0,34 & 0,02 & 0,58 & 10 & 0,85 & 0,59 \\
\hline Asteraceae & 1 & 1 & 1,96 & 0,11 & 0,03 & 0,08 & 10 & 0,28 & 0,16 \\
\hline
\end{tabular}

O destaque em número de indivíduos de Rubiaceae e Meliaceae, com espécies típicas do sub-bosque e subdossel, reflete o estágio de sucessão avançado do sub-bosque analisado, onde o sombreamento produzido pelo dossel de eucalipto deve estar favorecendo a regeneração dessas espécies tolerantes à sombra. Meliaceae é considerada indicativa da passagem de floresta pioneira em um estádio sucessional mais avançado (TABARELLI et al., 1994).

As espécies que apresentaram maiores valores de importância (VI) foram também as mais abundantes e dominantes, sendo elas: Psychotria sessilis (244), Siparuna guianensis (148), Erythroxylum pelleterianum (43), Miconia rigidiuscula (33), Miconia chamissois (28) e Carpotroche brasiliensis (26) (Quadro 4). Desse grupo, Psychotria sessilis, Siparuna guianensis e Carpotroche brasiliensis, que somaram $49,5 \%$ do total de indivíduos amostrados, regeneram em sub-bosques de florestas semidecíduas, sendo consideradas secundárias tardias, o que reflete também a importância da floresta de eucalipto como catalisadora de regeneração de espécies dos estádios sucessionais mais avançados. Essas espécies mais abundantes, além de serem típicas do grupo ecológico das secundárias tardias, têm também em comum a síndrome de dispersão de suas sementes, a zoocoria, o que tem possibilitado a ampla distribuição no sub-bosque de eucalipto a partir de fontes existentes na floresta semidecídua do entorno.

Considerando as síndromes de dispersão de sementes, 74\% (37) das espécies amostradas possuem dispersão zoocórica (Quadro 1). Esses resultados corroboram estudos realizados em florestas semidecíduas e de altitude da Serra do Japí, Jundiaí, SP, onde cerca de $90 \%$ das espécies do estrato de sub-bosque foram 
zoocóricas (MORELLATO e LEITÃO-FILHO, 1992). $\mathrm{Na}$ maioria das florestas tropicais, a zoocoria tem sido a principal forma de dispersão de sementes de espécies arbóreas e arbustivas (HOWE, 1990; MORELLATO eLEITÃO-FILHO, 1992; PINÃ-RODRIGUES eAGUIAR, 1993; MORELLATO, 1995).

Dada a importância da zoocoria para a dispersão de sementes de espécies arbustivo-arbóreas, tornase evidente que a proximidade de fontes dessas sementes seja igualmente um fator primordial para a regeneração florestal no sub-bosque de florestas plantadas. Povoamentos florestais homogêneos localizados próximos a fragmentos florestais tendem a ter mais rápida colonização do sub-bosque, bem como maior número de espécies do que plântios isolados dentro de grandes paisagens degradadas (KEENAN et al., 1997; LOUMETO e HUTTEL, 1997; PARROTA et al., 1997). Essa relação indica que a proximidade de fontes de propágulos é um dos principais fatores limitantes do processo de regeneração florestal em áreas degradadas, uma vez que a chuva de sementes é regulada pela densidade de indivíduos reprodutivos nas florestas próximas, regularidade da produção de sementes e disponibilidade de agentes dispersores, bem como da distância da fonte de propágulos (HARDWICK et al., 1997; WUNDERLE JR., 1997; RODRIGUES et al., 2004). Estudos sobre regeneração florestal no sub-bosque de florestas plantadas, realizados em povoamentos de Eucalyptus em diferentes regiões do Brasil (SCHILITTLER, 1984; SILVA JÚNIOR et al., 1995; CALEGARIO et al., 1993; SARTORI et al., 2002), indicam que essas florestas homogêneas podem realmente atuar como catalisadoras, facilitando a regeneração de espécies arbustivo-arbóreas nativas, e que a composição florística do sub-bosque é fortemente influenciada pela vegetação remanescente em seu entorno. Assim, tais florestas plantadas podem ser indicadas para a restauração florestal em áreas degradadas, técnica empregada com sucesso em Poços de Caldas, MG, com plantio de Mimosa scabrella Benth. (NAPPO et al., 2000).

Quadro 4 - Parâmetros fitossociológicos das espécies e do grupo das árvores mortas amostradas em sub-bosque de Eucalyptus grandis em Viçosa,MG, ordenadas de forma decrescente em valor de importância, em que: DA - densidade absoluta, DR - densidade relativa (\%), DoA - dominância absoluta (m²/ha), DoR - dominância relativa (\%), FA - freqüência absoluta, FR - freqüência relativa (\%) e VI - valor de importância (\%)

Table 4 - Estimate of phytosociological parameters of 50 species and group of dead trees sampled in in understory of Eucalyptus grandis in Viçosa, MG, in descending order of importance value, where DA - absolute density, DR - relative density (\%), DoA - absolute dominance $\left(\mathrm{m}^{2} / \mathrm{ha}\right)$, DoR - relative dominance (\%), FA - absolute frequency, $F R$ - relative frequency (\%), VI-importance value (\%)

\begin{tabular}{|c|c|c|c|c|c|c|c|}
\hline Espécies & DA & DR & DoA & DoR & FA & FR & VI \\
\hline Psychotria sessilis & 244 & 27,6 & 0,82 & 23,1 & 100 & 10 & 21 \\
\hline Siparuna guianensis Aubl. & 148 & 16,7 & 0,64 & 18,72 & 100 & 10 & 15 \\
\hline Erythroxylum pelleterianum A. St.-Hil. & 43 & 4,91 & 0,25 & 7,33 & 60 & 6,3 & 6,2 \\
\hline Grupo das mortas & 39 & 4,41 & 0,24 & 7,22 & 50 & 5,5 & 5,7 \\
\hline Vismia brasiliensis Choisy & 23 & 2,65 & 0,13 & 3,89 & 40 & 4,5 & 3,7 \\
\hline Alchornea glandulosa Poep. \& Endl. & 23 & 2,65 & 0,10 & 3,15 & 40 & 4,0 & 3,3 \\
\hline Miconia chamissois Naudin & 28 & 3,26 & 0,10 & 2,89 & 30 & 3,5 & 3,2 \\
\hline Miconia rigidiuscula Cogn. & 33 & 3,79 & 0,07 & 2,03 & 40 & 3,8 & 3,2 \\
\hline Carpotroche brasiliensis (Raddi) A. Gray & 26 & 2,99 & 0,07 & 2,11 & 40 & 3,8 & 2,9 \\
\hline Xylopia sericea A. St.-Hil. & 18 & 2,09 & 0,12 & 3,51 & 30 & 3,3 & 2,9 \\
\hline Anadenanthera colubrina (Vell.) Brenan & 23 & 2,69 & 0,01 & 0,48 & 40 & 3,8 & 2,3 \\
\hline Nectandra oppositifolia Nees \& Mart. & 18 & 2,01 & 0,07 & 2,27 & 20 & 2,0 & 2,1 \\
\hline Cabralea canjerana (Vell.) Mart. & 19 & 2,21 & 0,07 & 2,13 & 10 & 1,5 & 1,9 \\
\hline Piptadenia gonoacantha (Mart.) J.F. Macbr. & 16 & 1,85 & 0,03 & 0,93 & 30 & 3,0 & 1,9 \\
\hline Sparattosperma leucanthum (Vell.) K. Schum. & 11 & 1,22 & 0,06 & 1,73 & 20 & 2,5 & 1,8 \\
\hline Erythroxylum citrifolium A. St.-Hil. & 17 & 1,97 & 0,05 & 1,54 & 20 & 1,8 & 1,7 \\
\hline Solanum cernuиm Vell. & 12 & 1,45 & 0,02 & 0,68 & 20 & 2,3 & 1,4 \\
\hline Myrcia fallax (A. Richard) DC. & 8 & 0,93 & 0,02 & 0,82 & 20 & 2,0 & 1,3 \\
\hline Psychotria nuda (Cham. \& Schltdl.) Wawra & 12 & 1,46 & 0,01 & 0,36 & 20 & 2,0 & 1,3 \\
\hline
\end{tabular}


Quadro 4 - Cont

Table 4 - Cont.

\begin{tabular}{|c|c|c|c|c|c|c|c|}
\hline Espécies & $\mathrm{DA}$ & DR & DoA & DoR & FA & FR & VI \\
\hline Apuleia leiocarpa (Vogel) J.F. Macbr. & 8 & 0,93 & 0,03 & 0,87 & 10 & 1,5 & 1,1 \\
\hline Ocotea odorifera (Vellozo) Rohwer & 8 & 0,93 & 0,02 & 0,61 & 20 & 1,8 & 1,1 \\
\hline Copaifera langsdorffii Desf. & 6 & 0,73 & 0,04 & 1,23 & 10 & 1,3 & 1,1 \\
\hline Peltophorum dubium (Spreng.) Taub. & 6 & 0,73 & 0,02 & 0,86 & 10 & 1,5 & 1 \\
\hline Rollinia laurifolia Schltdl. & 7 & 0,83 & 0,02 & 0,69 & 10 & 1,5 & 1 \\
\hline Piper gaudichaudianum Kunth & 7 & 0,83 & 0,03 & 0,89 & 10 & 1,3 & 1 \\
\hline Psychotria longepedunculata (Gardner) Müll. Arg. & 8 & 0,93 & 0,01 & 0,48 & 10 & 1,5 & 1 \\
\hline Siparuna reginae (Tul.) A. DC. & 5 & 0,63 & 0,03 & 1,13 & 10 & 0,8 & 0,8 \\
\hline Trichilia lepidota Mart. & 8 & 0,93 & 0,01 & 0,38 & 10 & 1,0 & 0,8 \\
\hline Guatteria sellowiana Schltdl. & 6 & 0,73 & 0,06 & 0,21 & 10 & 1,3 & 0,7 \\
\hline Ilex brevicuspis Reissek & 5 & 0,65 & 0,03 & 0,74 & 10 & 0,8 & 0,7 \\
\hline Casearia decandra Jacq. & 5 & 0,65 & 0,02 & 0,48 & 10 & 1,0 & 0,7 \\
\hline Zanthoxylum rhoifolium Lam. & 3 & 0,38 & 0,03 & 0,93 & 10 & 0,8 & 0,7 \\
\hline Aspidosperma subincanum Mart. & 4 & 0,52 & 0,03 & 0,74 & 10 & 0,8 & 0,7 \\
\hline Matayba elaeagnoides Radlk. & 7 & 0,80 & 0,01 & 0,38 & 10 & 0,8 & 0,6 \\
\hline Pseudobombax grandiflorum (Cav.) A. Robyns & 3 & 0,38 & 0,03 & 0,76 & 10 & 0,8 & 0,6 \\
\hline Ocotea corymbosa (Meisn.) Mez & 4 & 0,52 & 0,02 & 0,54 & 10 & 0,8 & 0,6 \\
\hline Jacaranda macrantha Cham. & 2 & 0,24 & 0,02 & 0,66 & 10 & 0,5 & 0,5 \\
\hline Machaerium nyctitans (Vell.) Benth. & 2 & 0,24 & 0,02 & 0,52 & 10 & 0,5 & 0,4 \\
\hline Trichilia pallida $\mathrm{Sw} .$. & 2 & 0,24 & 0,01 & 0,39 & 10 & 0,5 & 0,4 \\
\hline Bauhinia forficata Link & 2 & 0,24 & 0,04 & 0,12 & 10 & 0,5 & 0,3 \\
\hline Cupania vernalis Cambess. & 4 & 0,52 & 0,04 & 0,12 & 10 & 0,3 & 0,3 \\
\hline \multicolumn{8}{|l|}{ Allophylus edulis (A. St.-Hil., } \\
\hline Cambess. \& A. Juss.) Radlk. & 2 & 0,24 & 0,02 & 0,06 & 10 & 0,5 & 0,3 \\
\hline Inga sessilis (Vell.) Mart. & 1 & 0,11 & 0,08 & 0,22 & 10 & 0,3 & 0,2 \\
\hline Dictyoloma incanescens DC. & 1 & 0,11 & 0,03 & 0,08 & 10 & 0,3 & 0,2 \\
\hline Vernonia diffusa Less. & 1 & 0,11 & 0,03 & 0,08 & 10 & 0,3 & 0,2 \\
\hline Machaerium aculeatum Raddi & 1 & 0,11 & 0,02 & 0,06 & 10 & 0,3 & 0,1 \\
\hline Casearia arborea (Rich.) Urb. & 1 & 0,11 & 0,02 & 0,06 & 10 & 0,3 & 0,1 \\
\hline Tapirira obtusa (Benth.) D.J. Mitch. & 1 & 0,11 & 0,02 & 0,05 & 10 & 0,3 & 0,1 \\
\hline Ocotea diffusa Van der Werff & 1 & 0,11 & 0,01 & 0,04 & 10 & 0,3 & 0,1 \\
\hline Rheedia gardneriana Planch. \& Triana & 1 & 0,11 & 0,07 & 0,02 & 10 & 0,3 & 0,1 \\
\hline Myrcia rostrata DC. & 1 & 0,11 & 0,03 & 0,01 & 10 & 0,3 & 0,1 \\
\hline
\end{tabular}

\section{CONCLUSÕES}

Comparando os índices de diversidade e eqüabilidade e a riqueza florística encontrados neste estudo com resultados obtidos em outros povoamentos florestais e em florestas semidecíduas, pode-se concluir que o povoamento de Eucalyptus grandis favoreceu a regeneração, em seu sub-bosque, de vegetação arbustivo-arbórea nativa, típica de florestas estacionais semideciduais da região. O plantio de espécies de rápido crescimento, como Eucalyptus grandis, pode ser uma alternativa de restauração florestal em áreas degradadas, em que a floresta plantada atua como catalisadora de regeneração de vegetação nativa no sub-bosque, desde que nas proximidades existam fragmentos florestais remanescentes.

\section{REFERÊNCIAS}

ARAÚJO, F. S. et al. Florística da vegetação arbustivo-arbórea colonizadora de uma área degradada por mineração de caulim, em Brás Pires, MG. Revista Árvore, v.29, n.6, p.107-116, 2005.

BERNACCI, L. C.; LEITÃO FILHO, H. F. Flora fanerogâmica da floresta da Fazenda São Vicente, Campinas, SP. Revista Brasileira de Botânica, v.19, p.149-164, 1996.

BRAZ, D.M.; CARVALHO-OKANO, R.M.; KAMEYAMA, C. Acanthaceae da Reserva Florestal Mata do Paraíso, Viçosa, Minas Gerais. Revista Brasileira de Botânica, v.25, p.495-504, 2002. 
BROWER, J. E.; ZAR, J. H. Field and laboratory methods for general ecology. 2.ed. Iowa: Wm. C. Brown Company, 1984. 226p.

CALEGARIO, N. et al. Parâmetros florísticos e fitossociologicos da regeneração natural de espécies arbóreas nativas no sub-bosque de povoamentos de Eucalyptus. Revista Árvore, v.17, n.1, p.16-29, 1993.

CAMPELLO, E. F. C. Sucessão vegetal na recuperação de áreas degradadas. In: DIAS, L. E.; MELlO, L.W.V. (Ed.) Recuperação de áreas degradadas. Viçosa, MG: Universidade Federal de Viçosa/SOBRADE, 1998. p.183-196.

CARVALHO, M. M. Recuperação de pastagens degradadas em áreas de relevo acidentado. In: DIAS, L. E.; MELLO, L. W. V. (Ed.)

Recuperação de áreas degradadas. Viçosa, MG: Universidade Federal de Viçosa/ SOBRADE, 1998, p.149-161.

CASTRO, P. S. et al. Interceptação da chuva por mata natural secundária na região de Viçosa, MG. Revista Árvore, v.7, p.76-89, 1983.

FAO. Efectos ecológicos de los eucaliptos. Roma: 1987. 106p.

GANDOLFI, S.; LEITÃO-FILHO, H. F.; BEZERRA, C. L. F. Levantamento florístico e caráter sucessional das espécies arbustivo-arbóreas de uma floresta mesófila semidecídua no município de Guarulhos, SP. Revista Brasileira de Biologia, v.55, n.4, p.753-767, 1995.

GELDENHUYS, C. J. Native forest regeneration in pine and eucalypt plantations in Northern Province, South África. Forest Ecology and Management, v.99, p.101-115, 1997.

GUEVARA, S.; PURATA, S. E.; van der MAAREL, E. The role of remnant forest trees in tropical secondary succession. Vegetation, v.66, p.77-84, 1986.

HARDWICK, K. et al. Understanding and assisting natural regeneration processes in degraded seasonal evergreen forests in northern Thailand. Forest Ecology and Management, v.99, p.203-214, 1997.
HOLL, K. D. Factors limiting tropical rain forest regeneration in abandoned pasture: seed rain, seed germination, microclimate, and soil. Biotropica, v.31, n.2, p.229-242, 1999.

HOWE, H. F. Seed dispersal by birds and mammals: implications for seedling demography. In: BAWA, K. S.; HADLEY, M. (Ed.) Reproductive ecology of tropical forest plants. Paris: UNESCO, 1990. p.191-218.

KEENAN, R.et al. Restoration of plant biodiversity beneath tropical tree plantations in Northern Australia. Forest Ecology and Management, v.99, p.117-131, 1997.

LIMA,W. P. Impacto ambiental do eucalipto. 2. ed. São Paulo: EDUSP, 1993. 301p.

LOUMETO, J. J.; HUTTEL, C. Understory vegetation in fast-growing tree plantations on savanna soils in Congo. Forest Ecology and Management, v.99, p.65-81, 1997.

MARTINS, S. V. et al. Colonization of gaps produced by death of bamboo clumps in a semideciduous mesophytic forest in south-eastern Brazil. Plant Ecology, v.172, p.12-131, 2004.

MARTINS, S. V.; RODRIGUES, R. R. Gap-phase regeneration in a semideciduous mesophytic forest, south-eastern Brazil. Plant Ecology, v.163, p.51-62, 2002.

MEIRA NETO, J. A. A. ; MARTINS, F. R. Estrutura da Mata da Silvicultura, uma floresta estacional semidecidual montana no município de Viçosa - MG. Revista Árvore, v.24, n.2, p.151-160, 2000.

MOBOT. Missouri Botanical Gardem. Disponível em (http://www.mobot.org/W3T/search/ vast.html), 2007.

MORELLATO, L. P. C.; LEITÃO FILHO, H. F. Padrões de frutificação e dispersão na Serra do Japí. In: MORELLATO, L. P. C. (Ed.) História natural da Serra do Japí: ecologia e preservação de uma área florestal no Sudeste do Brasil. Campinas: UNICAMP, FAPESP, 1992, p.112-137.

R. Árvore, Viçosa-MG, v.31, n.3, p.533-543, 2007 
MORELLATO, P. C. Frutos, frugívoros e a dispersão de sementes. In: MORELLATO, P. C., LEITÃO FILHO, H. F. (Ed.) Ecologia e preservação de uma floresta tropical urbana: Reserva de Santa Genebra.Campinas: UNICAMP, 1995. p.64-65.

MUELLER-DOMBOIS, D.; ELLENBERG, H. Aims and methods of vegetation ecology. New York: Willey \& Sons, 1974. 547p.

NAPPO, M. E.; OLIVEIRA FILHO, A. T.; MARTINS, S. V. A estrutura do sub-bosque de povoamentos homogêneos de Mimosa scabrella Bentham, em área minerada, em Poços de Caldas, MG. Ciência Florestal, v.10, n.2, p.17-29, 2000.

NERI, A. V. et al. Regeneração de espécies nativas lenhosas sob plantio de Eucalyptus em área de Cerrado na Floresta Nacional de Paraopeba, MG, Brasil. Acta Botânica Brasílica, v.19, n.2., p.369-376, 2005.

PARROTTA, J. A.; KNOWLES, O. H.; WUNDERLE JR., J. M. Development of floristic diversity in 10year-old restoration forests on bauxite mined site in Amazonia. Forest Ecology and

Management, v.99, p.21-42, 1997.

PINÃ-RODRIGUES, F. C. M.; AGUIAR, I. B. Maturação e dispersão de sementes. In: AGUIAR, I. B.; PIÑA-RODRIGUES, F. C. M.; FIGLIOLIA, M. B. (Ed.) Sementes florestais tropicais. Brasília: ABRATES, 1993. p.215-274.

POWERS, J. S.; HAGGAR, J. P.; FISHER, R. F. The effect of overstory composition on understory woody regeneration and species richness in 7-year-old plantations in Costa Rica. Forest Ecology and Management, v.99, p.43-54, 1997.

RIBAS, R. F. et al. Composição florística de dois trechos em diferentes etapas serais de uma Floresta Estacional Semidecidual em Viçosa, Minas Gerais. Revista Árvore, v.27, n.6, p.821-830, 2003.

RODRIGUES, R. R.; MARTINS, S. V.; BARROS, L. C. Tropical Rain Forest regeneration in an area degraded by mining in Mato Grosso State, Brazil. Forest Ecology and Management, v.90, p.323-333, 2004.

R. Árvore, Viçosa-MG, v.31, n.3, p.533-543, 2007
ROYAL BOTANIC GARDENS. Index

Kewensis on compacat disc - Manual. Oxford:University Press, 1993.67p.

SARTORI, M. S.; POGGIANI, F.; ENGEL, V. L. Regeneração da vegetação arbórea nativa no subbosque de um povoamento de Eucalyptus saligma Smith. localizado no Estado de São Paulo.

Scientia Forestalis, n.62, p.86-103, 2002.

SCHILITTLER, F. H. M. Composição florística e estrutura fitossociológica do sub-bosque de uma plantação de Eucalyptus tereticornis Sm., no município de Rio Claro, SP. 1984. 142f. Dissertação (Mestrado em Biologia Vegetal) - Universidade Estadual Paulista, Rio Claro, 1984.

SHEPHERD, G. J. Fitopac 1. Manual do usuário. Campinas: UNICAMP, 1994.

SILVA JÚNIOR, M. C.; SCARANO, F. R.; CARDEL, F. S. Regeneration of an Atlantic forest formation in the understorey of a Eucalyptus grandis plantation in south-eastern Brazil. Journal of Tropical Ecology, v.11, p.147-152, 1995.

SILVA, V. F.. et al. Caracterização estrutural de um fragmento de floresta semidecídua no município de Ibituruna, MG. Cerne, v. 9, n. 1, p. 95-110, 2003.

SILVA-JÚNIOR, W. M.et al. Regeneração natural de espécies arbustivo-arbóreas em dois trechos de uma Floresta Estacional Semidecidual, Viçosa, MG. Scientia Forestalis, n.66, p.69-79, 2004.

SOUZA, V. C.; LORENZI, H. Botânica sistemática: guia ilustrado para identificação das famílias de Angiospermas da flora brasileira, baseado em APG II. Nova Odessa: Instituto Plantarum, 2005.

TABARELLI, N.; VILLANI, J. P.; MANTOVANI, W. Estudo comparativo da vegetação de dois trechos de floresta secundária no Núcleo Santa Virginia, Parque Estadual da Serra do Mar, SP. Revista do Instituto Florestal, v.6, p.1-11, 1994.

van der PIJL, L. Principles of dispersal in higher plants. New York: Springer-Verlag, 1982. 214p. 
VELOSO, H. P.; RANGEL FILHO, A. L. R.; LIMA, J. C. A. Classificação da vegetação brasileira adaptada a um sistema universal. Rio de Janeiro: Instituto Brasileiro de Geografia e Estatística, 1991.
WUNDERLE JR., M. J. The role of animal seed dispersal in accelerating native forest regeneration on degraded tropical lands. Forest Ecology and Management, v.99, p.223-235, 1997. 
